\title{
The role of bronchoscopy in patients with SARS-CoV-2
}

\section{pneumonia}

\author{
Marisol Arenas-De Larriva ${ }^{1}$, Roberto Martín-DeLeon $\mathbb{B}^{2}$, Blanca Urrutia Royo ${ }^{3}$, Iker Fernández-Navamuel ${ }^{4}$, \\ Andrés Gimenez Velando ${ }^{4}$, Laura Nuñez García ${ }^{4}$, Carmen Centeno Clemente ${ }^{5}$, Felipe Andreo García $\mathbb{D}^{5}$, \\ Albert Rafecas Codern ${ }^{6}$, Carmen Fernández-Arias ${ }^{6}$, Virginia Pajares Ruiz ${ }^{6}$, Alfons Torrego Fernández ${ }^{6}$, \\ Olga Rajas ${ }^{7}$, Gorane Iturricastillo ${ }^{8}$, Ricardo Garcia Lujan ${ }^{9}$, Lorena Comeche Casanova ${ }^{10}$, \\ Albert Sánchez-Font ${ }^{11}$, Ricardo Aguilar-Colindres ${ }^{12}$, Roberto Larrosa-Barrero ${ }^{13}$, Ruth García García ${ }^{14}$, \\ Rosa Cordovilla ${ }^{14}$, Ana Núñez-Ares ${ }^{15}$, Andrés Briones-Gómez ${ }^{16}$, Enrique Cases Viedma ${ }^{16}$, José Franco ${ }^{17}$, \\ Javier Cosano Povedano ${ }^{18}$, Manuel Luis Rodríguez-Perálvarez $\mathbb{C}^{19}$, Jose Joaquin Cebrian Gallardo ${ }^{20}$, \\ Manuel Nuñez Delgado ${ }^{21}$, María Pavón-Masa ${ }^{22}$, Maria del Mar Valdivia Salas ${ }^{23}$ and Javier Flandes $\mathbb{B}^{24}$
}

\begin{abstract}
${ }^{1}$ Dept of Bronchoscopy and Interventional Pulmonology, Hospital Universitario Reina Sofía, IMIBIC, Córdoba, Spain. ${ }^{2}$ Dept of Pulmonology, Hospital Universitario Reina Sofía, IMIBIC, Córdoba, Spain. ${ }^{3}$ Pulmonary Dept, Thorax Clinic Institute, Hospital Universitari Germans Trias i Pujol, Badalona, Spain. ${ }^{4}$ Bronchoscopy and Interventional Pulmonology Unit, Pulmonology Dept, Hospital Fundacion Jimenez Diaz, ISS-FJD, CIBERES, Madrid, Spain. ${ }^{5}$ Interventional Pulmonology Unit, Pulmonary Dept, Thorax Clinic Institute, Hospital Universitari Germans Trias i Pujol, UAB, IGTP, Badalona, Spain. ${ }^{6}$ Respiratory Dept, Hospital Santa Creu i Sant Pau, Barcelona, Spain. ${ }^{7}$ Interventional Pulmonology Unit, Pulmonology Dept, Hospital Universitario de la Princesa, Instituto de Investigación Princesa, Madrid, Spain. ${ }^{8}$ Pulmonology Dept, Hospital Universitario de la Princesa, Instituto de Investigación Princesa, Madrid, Spain. ${ }^{9}$ Dept of Interventional Pulmonology, Hospital Universitario 12 Octubre and Hospital Univesitario Quirónsalud Madrid, Madrid, Spain. ${ }^{10}$ Dept of Pulmonology, Hospital Universitario Quirónsalud Madrid, Madrid, Spain. ${ }^{11}$ Pulmonology Dept, Hospital del Mar, CIBERES, UAB, IMIM (Hospital del Mar Medical Research Institute), Barcelona, Spain. ${ }^{12}$ Pulmonology Dept, Hospital del Mar, CIBERES, Barcelona, Spain. ${ }^{13}$ Pulmonary Dept, Hospital Universitario Clínico San Carlos, Madrid, Spain. ${ }^{14}$ Interventional Pulmonology Unit, Pulmonary Dept, Salamanca University Hospital, Salamanca, Spain. ${ }^{15}$ Interventional Pulmonology Unit, Pulmonary Dept, Albacete, Spain. ${ }^{16}$ Interventional Pulmonology Unit, Pulmonary Dept, Hospital Universitario y Politécnico La Fe, Valencia, Spain. ${ }^{17}$ Pneumology Service, Clinic University Hospital, INCLIVA Health Research Institute, Valencia, Spain. ${ }^{18}$ Bronchoscopy and Interventional Pulmonology Unit, Pulmonology Dept, Hospital Universitario Reina Sofía, IMIBIC, Córdoba, Spain. ${ }^{19}$ Maimónides Institute of Biomedical Research of Córdoba (IMIBIC), CIBERehd, Córdoba, Spain. ${ }^{20}$ Dept of Pulmonology, Agencia sanitaria Costa del Sol, Marbella, Spain. ${ }^{21}$ Dept of Bronchoscopy and Interventional Pulmonology, Hospital Álvaro Cunqueiro, CHUVI, Vigo, Spain. ${ }^{22}$ Dept of Interventional Pulmonology, Hospital Universitario Virgen Macarena, Seville, Spain. ${ }^{23}$ Dept of Interventional Pulmonology, Hospital Universitario Santa Lucía, Cartagena, Spain. ${ }^{24}$ Bronchoscopy and Interventional Pulmonology Unit, Pulmonology Dept, Hospital Fundación Jimenez Diaz, ISS-FJD, CIBERES, Madrid, Spain.
\end{abstract}

Corresponding author: Marisol Arenas-De Larriva (arlam23@hotmail.com)

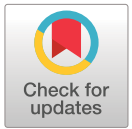

Copyright @The authors 2021

This version is distributed under the terms of the Creative Commons Attribution NonCommercial Licence 4.0. For commercial reproduction rights and permissions contact permissions@ersnet.org
Shareable abstract (@ERSpublications)

Bronchoscopy is part of the armamentarium against \#COVID19. It allows diagnosis, facilitates mechanical ventilation and provides prognostic information. This information could be used to refine healthcare pathways in order to improve outcomes. https://bit.ly/2QuAQOt

Cite this article as: Arenas-De Larriva M, Martín-DeLeon R, Urrutia Royo B, et al. The role of bronchoscopy in patients with SARS-CoV-2 pneumonia. ERJ Open Res 2021; 7: 00165-2021 [DOI: 10.1183/23120541.00165-2021].

\section{Abstract}

Background The role of bronchoscopy in coronavirus disease 2019 (COVID-19) is a matter of debate. Patients and methods This observational multicentre study aimed to analyse the prognostic impact of bronchoscopic findings in a consecutive cohort of patients with suspected or confirmed COVID-19. Patients were enrolled at 17 hospitals from February to June 2020. Predictors of in-hospital mortality were assessed by multivariate logistic regression.

Results A total of 1027 bronchoscopies were performed in 515 patients (age $61.5 \pm 11.2$ years; 73\% men), stratified into a clinical suspicion cohort $(n=30)$ and a COVID-19 confirmed cohort $(n=485)$. In the clinical suspicion cohort, the diagnostic yield was 36.7\%. In the COVID-19 confirmed cohort, bronchoscopies were predominantly performed in the intensive care unit $(n=961 ; 96.4 \%)$ and major indications were: difficult mechanical ventilation (43.7\%), mucus plugs (39\%) and persistence of radiological infiltrates (23.4\%). 
This article has supplementary material available from openres.ersjournals.com

Received: 9 March 2021 Accepted: 22 April 2021
147 bronchoscopies were performed to rule out superinfection, and diagnostic yield was $42.9 \%$. There were abnormalities in $91.6 \%$ of bronchoscopies, the most frequent being mucus secretions (82.4\%), haematic secretions (17.7\%), mucus plugs (17.6\%), and diffuse mucosal hyperaemia (11.4\%). The independent predictors of in-hospital mortality were: older age (OR 1.06; $p<0.001$ ), mucus plugs as indication for bronchoscopy (OR 1.60; $\mathrm{p}=0.041$ ), absence of mucosal hyperaemia (OR 0.49; $\mathrm{p}=0.041$ ) and the presence of haematic secretions (OR 1.79; $\mathrm{p}=0.032$ ).

Conclusion Bronchoscopy may be indicated in carefully selected patients with COVID-19 to rule out superinfection and solve complications related to mechanical ventilation. The presence of haematic secretions in the distal bronchial tract may be considered a poor prognostic feature in COVID-19.

\section{Introduction}

The novel coronavirus disease 2019 (COVID-19), which is caused by the severe acute respiratory syndrome coronavirus 2 (SARS-CoV-2), originated in Wuhan in the province of Hubei, China, in December 2019 [1]. COVID-19 rapidly spread to other countries driven by an increased prevalence of asymptomatic carriers and by the airborne transmission of SARS-CoV-2 [2]. In March 2020, COVID-19 was declared a pandemic by the World Health Organization, and since then has challenged healthcare systems worldwide, making the need to optimise clinical pathways and resource utilisation mandatory.

The role of bronchoscopy in COVID-19 is a matter of debate. Among patients with clinical suspicion of COVID-19 with negative nasopharyngeal swab specimen results by real-time PCR with reverse transcription (RT-PCR), bronchoscopy could provide increased sensitivity by obtaining samples from the lower respiratory tract [3]. In patients with severe COVID-19, mainly admitted to the intensive care unit (ICU), bronchoscopy may be required to manage complications such as atelectasis or haemoptysis, to solve issues with mechanical ventilation, and to rule out superinfection. However, bronchoscopy in COVID-19 is not without risks, including disease transmission to healthcare staff. Although some scientific societies have issued guidelines in order to reduce heterogeneity in clinical practice [4], the supporting scientific background is scarce and is mainly composed by short series [5-7].

The main end-point of the present nationwide study was to evaluate the impact of endoscopic findings on outcomes among patients with COVID-19. Secondary outcomes were: 1) to describe the indications for bronchoscopy and procedures; 2) to analyse the diagnostic yield of bronchoscopy in patients with suspected SARS-CoV-2 pneumonia.

Materials and methods

The “COronavirus \& BRonchoscopy in Spain (COBRE)" project is an ambispective multicentre study, which was launched during the first epidemic wave of COVID-19 in Spain. The study was performed according to the principles of the Declaration of Helsinki and aligning with the European Union regulation 2016/679. The study was approved by the Research Ethics Committee of the Hospital Universitario Reina Sofía, Córdoba, Spain (PI 2020/4680).

\section{Study population}

Patients were enrolled at 17 secondary and tertiary hospitals in Spain. The recruitment period ranged from February 20, 2020, when the national authorities informed about community transmission of SARS-CoV-2, until June 30, 2020, when there was an official declaration of controlled community transmission. Patients admitted to the hospital because of suspected or confirmed COVID-19 who required a bronchoscopy were consecutively included and stratified into two study cohorts:

1. Clinical suspicion cohort: patients with clinical and radiological features of COVID-19 or positive IgM antibody testing, but without confirmation by RT-PCR in two consecutive nasopharyngeal swab specimens, who underwent bronchoscopy for diagnostic purposes.

2. RT-PCR confirmed cohort: patients with SARS-CoV-2 pneumonia confirmed by RT-PCR of nasopharyngeal swab specimens who required a bronchoscopy.

The exclusion criteria for both cohorts were as follows: patients younger than 18 years old; bronchoscopy performed after virological resolution (confirmed by two consecutive RT-PCR negative tests); interval between COVID-19 confirmation and endoscopic examination longer than 30 days.

\section{Identification of study candidates, data extraction and outcomes}

Potential study candidates were screened among patients admitted to the hospital with suspected or confirmed COVID-19. Those patients with compatible clinical symptoms and typical radiological findings [8] with two negative RT-PCR of nasopharyngeal swab specimens could undergo bronchoscopy to obtain a lower respiratory tract specimen and they formed the clinical suspicion cohort. Patients with previous 
positive RT-PCR of SARS-CoV-2 in nasopharyngeal swab specimens who underwent bronchoscopy to rule out superinfection or for therapeutic purposes formed the RT-PCR-confirmed cohort.

Data were recorded in an anonymised electronic datasheet using the REDCap (Research Electronic Data Capture) platform [9]. Study investigators received online training at baseline to homogenise the data collection, and they were granted access with a unique username/password. All clinical information was extracted from reliable electronic medical data sources. Demographic characteristics and comorbidities (graded with the Charlson comorbidity index as absent if $0-1$, mild if 2 or severe if $\geqslant 3$ [10]), clinical symptoms and diagnostic tests of COVID-19 were recorded. Blood tests and radiological features were considered within the $48 \mathrm{~h}$ prior to bronchoscopy. Imaging findings obtained in chest computed tomography (CT) were reported according to the COVID-RADS classification as typical, fairly typical, atypical or normal [11]. Bronchoscopic findings and procedures were also registered. Patients were followed until hospital discharge or death. The main outcome evaluated was in-hospital mortality at 90 days after bronchoscopy.

\section{Sample size calculation}

The sample size was calculated using EPIDAT version 4.2 (Xunta de Galicia, Spain). The following assumptions were made to study a theoretical relationship between endoscopic findings and outcomes:

- The prevalence of an endoscopic feature indicating poor prognosis: $20 \%$.

- In-hospital mortality in patients showing an endoscopic feature indicating poor prognosis: $40 \%$ (obtained from the upper range of mortality reported in previous series of critically ill patients [12, 13]).

- In-hospital mortality in patients without an endoscopic feature indicating poor prognosis: $25 \%$ (obtained from the lower range of mortality reported in previous series of critically ill patients [12, 13]).

- $\quad$ Statistical power: $80 \%$

- $\alpha$ error: $5 \%$

- Incomplete or unavailable data: $5 \%$

Under these premises, the minimum sample size required was 483 patients with RT-PCR-confirmed COVID-19. The study finally comprised 515 patients, including 488 RT-PCR-confirmed cases.

\section{Statistical analysis}

Categorical variables were described as frequency tables and percentages. Continuous variables were described using mean and standard deviation, except for those with an asymmetric distribution, in which median and interquartile range (IQR) were used. To identify clinical, radiological and endoscopic features associated with in-hospital mortality at 90 days, the first bronchoscopy performed in each patient with RT-PCR-confirmed COVID-19 was considered. Univariate and multivariate logistic regression was used. Variables with $\mathrm{p}<0.30$ in the univariate analysis were entered the initial multivariate model. Endoscopic features with a prevalence $\geqslant 5 \%$ were also included in the initial multivariate model irrespective of their univariate p-value. Nonsignificant covariates were removed in a backward stepwise process. All possible interactions were tested. Clinically meaningful variables were also kept in the final model even if they did not reach statistical significance. Kaplan-Meier curves were used for survival analysis, being patients censored at hospital discharge or on October 30, 2020. The statistical analysis was performed using SPSS version 22.0 (IBM Corp, Armonk, NY, USA). Every hypothesis tested was two-tailed and considered significant if $\mathrm{p}<0.05$.

\section{Results}

Description of the study population

A total of 1027 bronchoscopies were performed in 515 patients (average age $61.5 \pm 11.2$; 73\% men). The clinical suspicion cohort comprised 30 patients (5.8\%), while the remaining 485 patients (94.2\%) were RT-PCR-confirmed COVID-19, including 86 patients who underwent 147 bronchoscopies to rule out superinfection and 399 patients who required 850 therapeutic bronchoscopies. The clinical characteristics of both cohorts are summarised in table 1 . Severe comorbidity defined as a Charlson score $\geqslant 3$ was more frequent in the clinical suspicion cohort (33.3\%) as compared with the RT-PCR-confirmed cohort (10.1\%) $(\mathrm{p}<0.001)$. The clinical presentation was almost indistinguishable in both cohorts, except for an increased prevalence of cough and myalgias in the RT-PCR-confirmed cohort $(74.2 \%$ versus $50 \%, \mathrm{p}=0.004$; and $32.2 \%$ versus $13.3 \%, \mathrm{p}=0.031$, respectively). In the radiographs, bilateral infiltrates predominated in the RT-PCR-confirmed cohort (83.5\% versus $60 \%$; $\mathrm{p}<0.001$ ). Admission to the ICU was required in $95.2 \%$ of patients in the RT-PCR-confirmed cohort as compared with $26.7 \%$ of patients in the clinical suspicion cohort $(\mathrm{p}<0.001)$. 


\begin{tabular}{|c|c|c|c|}
\hline Variable & $\begin{array}{l}\text { Clinical suspicion } \\
\text { cohort }(n=30)\end{array}$ & $\begin{array}{l}\text { RT-PCR-confirmed } \\
\text { cohort }(n=485)\end{array}$ & p-value \\
\hline Age, mean \pm SD & $59.2 \pm 15.5$ & $61.7 \pm 10.9$ & 0.390 \\
\hline Women & $36.7 \%(11)$ & $26.4 \%(128)$ & 0.219 \\
\hline \multicolumn{4}{|l|}{ Previous medical history } \\
\hline Diabetes & $20 \%(6)$ & $22.5 \%(109)$ & 0.752 \\
\hline Hypertension & $36.7 \%(11)$ & $47.6 \%(231)$ & 0.243 \\
\hline Cardiovascular & $13.3 \%(4)$ & $10.9 \%(53)$ & 0.684 \\
\hline Bronchopulmonary & $23.3 \%(7)$ & $14 \%(68)$ & 0.161 \\
\hline Neoplasms & $30 \%(9)$ & $9.3 \%(45)$ & 0.002 \\
\hline Charlson comorbidity index & & & $<0.001$ \\
\hline $0-1$ & $53.3 \%(16)$ & $77.3 \%(375)$ & \\
\hline 2 & $13.3 \%(4)$ & $12.6 \%(61)$ & \\
\hline$\geqslant 3$ & $33.3 \%(10)$ & $10.1 \%(49)$ & \\
\hline Tobacco consumption & & & 0.046 \\
\hline Current smokers & $17.9 \%(5)$ & $6.2 \%(29)$ & \\
\hline Past smokers & $17.9 \%(5)$ & $28 \%(130)$ & \\
\hline Nonsmokers & $64.2 \%(18)$ & $65.8 \%(306)$ & \\
\hline $\begin{array}{l}\text { Lifetime tobacco consumption", } \\
\text { pack-years }\end{array}$ & $21.5(11.5-46.2)$ & $30(15-40)$ & 0.988 \\
\hline \multicolumn{4}{|l|}{ Immunosuppression } \\
\hline HIV & $3.3 \%(1)$ & $0.8 \%(4)$ & 0.174 \\
\hline Chemotherapy & $16.7 \%(5)$ & $1 \%(5)$ & $<0.001$ \\
\hline Monoclonal antibodies & $3.3 \%(1)$ & $1 \%(5)$ & 0.304 \\
\hline Calcineurin inhibitors & $10 \%(3)$ & $2.1 \%(10)$ & 0.034 \\
\hline Antimetabolites & $6.7 \%(2)$ & $2.1 \%(10)$ & 0.151 \\
\hline Corticosteroids & $0 \%(0)$ & $2.9 \%(14)$ & 1 \\
\hline \multicolumn{4}{|l|}{ Clinical presentation of COVID-19 } \\
\hline Fever & $76.7 \%(23)$ & $83.1 \%(403)$ & 0.366 \\
\hline Dyspnoea & $56.7 \%(17)$ & $67.4 \%(327)$ & 0.225 \\
\hline Cough & $50 \%(15)$ & $74.2 \%(360)$ & 0.004 \\
\hline Gastrointestinal symptoms & $16.7 \%(5)$ & $23.1 \%(112)$ & 0.415 \\
\hline Myalgias & $13.3 \%(4)$ & $32.2 \%(156)$ & 0.031 \\
\hline Anosmia/ageusia & $0(0 \%)$ & $6.6 \%(32)$ & 0.245 \\
\hline \multicolumn{4}{|l|}{ Laboratory parameters } \\
\hline$P_{\mathrm{aO}_{2}} / F_{\mathrm{IO}_{2}}$ ratio & $270(196-288)$ & $160(118-216)$ & 0.038 \\
\hline $\mathrm{S}_{\mathrm{aO}_{2}} / F_{\mathrm{IO}_{2}}$ ratio $^{+}$ & $329(235-387)$ & $184(132-239)$ & $<0.001$ \\
\hline Lymphocyte count $\cdot \mu \mathrm{L}^{-1}$ & $890(490-1540)$ & $700(540-1000)$ & 0.115 \\
\hline D dimer $\mathrm{ng} \cdot \mathrm{mL}^{-1}$ & $1113(577-2170)$ & $843(492-1605)$ & 0.545 \\
\hline Lactate dehydrogenase $\mathrm{U} \cdot \mathrm{L}^{-1}$ & $304(239-507)$ & $450(340-625)$ & 0.049 \\
\hline Ferritin $\mathrm{ng} \cdot \mathrm{mL}^{-1}$ & $589(359-1356)$ & $1275(648-2299)$ & 0.107 \\
\hline C-reactive protein $\mathrm{mg} \cdot \mathrm{L}^{-1}$ & $36(12-166)$ & $22(11-81)$ & 0.438 \\
\hline Interleukin-6 pg·mL $\mathrm{m}^{-1}$ & $46(5-149)$ & $65(23-130)$ & 0.546 \\
\hline Chest radiograph abnormalities & & & $<0.001$ \\
\hline Normal & $0 \%(0)$ & $0.4 \%(2)$ & \\
\hline Unilateral interstitial & $23.3 \%(7)$ & $1.6 \%(8)$ & \\
\hline Bilateral interstitial & $43.3 \%(13)$ & $36.7 \%(178)$ & \\
\hline Unilateral consolidation & $6.7 \%(2)$ & $2.9 \%(14)$ & \\
\hline Bilateral consolidation & $16.7 \%(5)$ & $46.8 \%(227)$ & \\
\hline Others & $10 \%(3)$ & $11.5 \%(56)$ & \\
\hline \multicolumn{4}{|l|}{ COVID-19-specific therapy } \\
\hline Azithromycin & $0 \%(0)$ & $50.4 \%(242)$ & $<0.001$ \\
\hline Hydroxychloroquine & $3.3 \%(1)$ & $75.4 \%(362)$ & $<0.001$ \\
\hline Lopinavir/ritonavir & $3.3 \%(1)$ & $54 \%(259)$ & $<0.001$ \\
\hline Remdesivir & $0 \%(0)$ & $5 \%(24)$ & 0.386 \\
\hline Interferon- $\beta$ & $3.3 \%(1)$ & $15.2 \%(73)$ & 0.104 \\
\hline Anakinra & $0 \%(0)$ & $3.8 \%(18)$ & 0.616 \\
\hline Tocilizumab & $3.3 \%(1)$ & $49.2 \%(236)$ & $<0.001$ \\
\hline Antibiotics & $3.3 \%(1)$ & $31.7 \%$ (152) & $<0.001$ \\
\hline
\end{tabular}




\begin{tabular}{|c|c|c|c|}
\hline Variable & $\begin{array}{l}\text { Clinical suspicion } \\
\text { cohort }(n=30)\end{array}$ & $\begin{array}{l}\text { RT-PCR-confirmed } \\
\text { cohort }(n=485)\end{array}$ & $\mathrm{p}$-value \\
\hline Corticosteroids & $3.3 \%(1)$ & $70.4 \%(338)$ & $<0.001$ \\
\hline Length of hospital stay, days & $18(8-28)$ & $38(22-61)$ & 0.007 \\
\hline Admission to intensive care unit & $26.7 \%(8)$ & $95.2 \%(456)$ & $<0.001$ \\
\hline In-hospital mortality & $20 \%(6)$ & $33.6 \%(163)$ & 0.123 \\
\hline \multicolumn{4}{|c|}{$\begin{array}{l}\text { Data are presented as } \%(\mathrm{n}) \text { or median (interquartile range), unless otherwise stated. } P_{\mathrm{aO}_{2}}: \text { arterial oxygen } \\
\text { tension; } F_{\mathrm{IO}_{2}}: \text { inspiratory oxygen fraction; } S_{\mathrm{aO}_{2}}: \text { arterial oxygen saturation. }{ }^{\#}: \text { only accounted for current/past } \\
\text { smokers; }{ }^{\mathrm{I}}: \mathrm{P}_{\mathrm{aO}_{2}} / F_{\mathrm{IO}_{2}} \text { was available in } 298 \text { patients; }{ }^{+}: S_{\mathrm{aO}_{2}} / F_{\mathrm{IO}_{2}} \text { was available in } 140 \text { patients who did not have } \\
P_{\mathrm{aO}_{2}} / F_{\mathrm{IO}_{2}} .\end{array}$} \\
\hline
\end{tabular}

\section{Clinical suspicion cohort}

Bronchoscopies were performed in the bronchoscopy room (50\%), ICU (26.7\%), respiratory ward (20\%) or in the operating room (3.3\%). Disposable bronchoscopes were used in 18 procedures (60\%) and the preferred access was via nasal (63.3\%). Lower respiratory tract specimens obtained were: bronchial aspiration (BAS) (31.6\%), bronchoalveolar lavage (BAL) (10.5\%), bronchial washing (10.5\%) and a combination of BAS and BAL (47.4\%). RT-PCR was positive for SARS-CoV-2 in 11 patients (36.7\%). Of note, none of the patients undergoing BAS alone had a positive RT-PCR, while the diagnostic yield of the remaining specimens ranged from $40 \%$ to $60 \%$. Among 19 patients without confirmation of SARS-CoV-2, 5 patients (26.3\%) had an alternative diagnosis (Cytomegalovirus, Pneumocystis, Aspergillus and/or Staphylococcus), and 14 patients had no proven microbiological agent in the lower respiratory tract specimens. None of these patients had a subsequent positive test for COVID-19. Patients with and without SARS-CoV-2 confirmation did not show statistical differences regarding age $(p=0.90)$, sex distribution $(\mathrm{p}=0.70)$, smoking history $(\mathrm{p}=0.18)$ and Charlson comorbidity index $(\mathrm{p}=0.47)$. Fever, cough, dyspnoea and myalgias were distributed homogeneously in both groups $(\mathrm{p}=0.61, \mathrm{p}=0.70, \mathrm{p}=0.13$, and $\mathrm{p}=0.61$, respectively). Patients with a SARS-CoV-2-positive RT-PCR were characterised by an increased prevalence of gastrointestinal symptoms (36.4\% versus $5.3 \%$; $\mathrm{p}=0.047$ ). Laboratory parameters including lymphocyte count, D dimer, lactate dehydrogenase, ferritin, C-reactive protein and interleukin-6 were similar in the RT-PCR-positive and -negative groups (data not shown). The chest radiographs showed interstitial bilateral infiltrates in $63.6 \%$ of patients from the SARS-CoV-2-positive group as compared with $31.6 \%$ of patients without COVID-19 confirmation ( $p=0.09$ ). A chest CT was performed in 14 patients within $48 \mathrm{~h}$ prior to bronchoscopy (6 patients with subsequent positive RT-PCR and eight patients with subsequent negative RT-PCR). There was a typical or fairly typical radiological pattern of COVID-19 in the vast majority of patients (78.6\%), without statistical differences between patients with subsequent positive and negative RT-PCR results. There were endoscopic abnormalities in $63.6 \%$ of patients with positive COVID-19 RT-PCR versus 36.8\% of patients with negative COVID-19 RT-PCR results $(\mathrm{p}=0.16)$. The most frequent bronchoscopic findings were thick mucus secretion ( $\mathrm{n}=9$ ), fluid mucus secretion $(n=4)$ and diffuse mucosal hyperaemia $(n=3)$. Admission to the ICU was required in $18.2 \%$ of patients with a positive RT-PCR and in $31.6 \%$ of patients with a negative RT-PCR ( $\mathrm{p}=0.67)$. The in-hospital mortality was $18.2 \%$ in patients with a SARS-CoV-2-positive RT-PCR and $21.1 \%$ in patients with negative RT-PCR (log-rank p=0.47).

\section{RT-PCR-confirmed cohort}

The RT-PCR-confirmed cohort included 485 hospitalised patients who underwent 997 bronchoscopies (range 1-16 procedures per patient). The number of healthcare professionals involved in each procedure ranged from 1 to 5. Bronchoscopies were performed predominantly in the ICU ( $n=961 ; 96.4 \%)$, followed by the COVID-19 ward ( $n=18 ; 1.8 \%)$, endoscopy room $(n=15 ; 1.5 \%)$ and operating room $(n=3 ; 0.3 \%)$. The vast majority of procedures were performed in rooms without negative pressure (90.7\%) and using disposable bronchoscopes (94.5\%). Regarding ventilatory support, most bronchoscopies were performed with patients under invasive mechanical ventilation (93.2\%) and in 66 cases (6.6\%) under extracorporeal membrane oxygenation. The predominant accesses were orotracheal tube (61\%) and tracheostomy (35.2\%). The patient was in prone position in 55 bronchoscopies (5.5\%). The ratio of partial pressure arterial oxygen and fraction of inspired oxygen was $171.9 \pm 80.6$. Bronchoscopies were indicated to rule out superinfection $(14.7 \%)$ or for therapeutic purposes (85.3\%). Therapeutic indications and endoscopic findings are summarised in table 2. Major indications for bronchoscopy were complications associated with mechanical ventilation (50\%), mucus plugs/atelectasis (46\%), persistence or progression of radiological infiltrates (33.4\%) and haemoptysis (6\%). There were endoscopic abnormalities in 91.6\%, the 
TABLE 2 Therapeutic indications and findings in 997 bronchoscopies performed in 485 hospitalised patients with RT-PCR-confirmed SARS-CoV-2 pneumonia

\begin{tabular}{|c|c|}
\hline \multicolumn{2}{|l|}{ Indications } \\
\hline Atelectasis & $7 \%(70)$ \\
\hline Mucus plugs & $39 \%(389)$ \\
\hline Haemoptysis & $6 \%(60)$ \\
\hline Radiological progression & $10 \%(100)$ \\
\hline Persistence of radiological infiltrates & $23.4 \%(233)$ \\
\hline Difficult mechanical ventilation & $43.7 \%(436)$ \\
\hline Impossible weaning from mechanical ventilation & $6.3 \%(63)$ \\
\hline \multicolumn{2}{|l|}{ Findings } \\
\hline Normal & $8.4 \%(84)$ \\
\hline Diffuse mucosal hyperaemia & $11.4 \%(114)$ \\
\hline Thick mucus secretion & $59.9 \%(597)$ \\
\hline Fluid mucus secretion & $22.5 \%(224)$ \\
\hline Mucus plugs & $17.6 \%(175)$ \\
\hline Haematic secretions & $17.7 \%(176)$ \\
\hline Intrabronchial clots & $6 \%(60)$ \\
\hline \multicolumn{2}{|l|}{ Location of mucus plugs ( $n=175$ ) } \\
\hline Trachea & $24 \%(42)$ \\
\hline Main right bronchus & $31.4 \%(55)$ \\
\hline Main left bronchus & $33.5 \%(59)$ \\
\hline Right superior bronchus & $18.3 \%(32)$ \\
\hline Right middle bronchus & $24 \%(42)$ \\
\hline Right inferior bronchus & $45.1 \%(79)$ \\
\hline Left superior bronchus & $16 \%(28)$ \\
\hline Left inferior bronchus & $36.6 \%(64)$ \\
\hline \multicolumn{2}{|l|}{ Location of intrabronchial clots $(n=60)$} \\
\hline Trachea & $31.7 \%(19)$ \\
\hline Main right bronchus & $55 \%(33)$ \\
\hline Main left bronchus & $41.7 \%(25)$ \\
\hline Right superior bronchus & $15 \%(9)$ \\
\hline Right middle bronchus & $21.7 \%(13)$ \\
\hline Right inferior bronchus & $40 \%(24)$ \\
\hline Left superior bronchus & $10 \%(6)$ \\
\hline Left inferior bronchus & $20 \%(12)$ \\
\hline \multicolumn{2}{|l|}{ Therapy } \\
\hline Aspiration & $82.3 \%(821)$ \\
\hline Removal with grasp forceps & $1.4 \%(14)$ \\
\hline Cannula placement & $0.3 \%(3)$ \\
\hline Bronchial occlusion & $0.2 \%(2)$ \\
\hline Cryotherapy & $0.1 \%(1)$ \\
\hline Endobronchial selective intubation & $0.1 \%(1)$ \\
\hline \multicolumn{2}{|l|}{ Intrabronchial drugs } \\
\hline Saline solution & $60.2 \%(600)$ \\
\hline Mesna & $5.1 \%(51)$ \\
\hline Hypertonic solution & $14.5 \%(145)$ \\
\hline $\mathrm{N}$-acetylcysteine & $6 \%(60)$ \\
\hline Hyaluronic acid (+hypertonic solution) & $6.5 \%(65)$ \\
\hline Others & $0.9 \%(9)$ \\
\hline \multicolumn{2}{|l|}{ Samples } \\
\hline Bronchial aspiration & $43 \%(429)$ \\
\hline Combined bronchial aspiration and bronchoalveolar lavage & $24.3 \%(242)$ \\
\hline Bronchoalveolar lavage & $5.8 \%(58)$ \\
\hline Bronchial washing & $11 \%(110)$ \\
\hline \multicolumn{2}{|l|}{ Microbiological agents } \\
\hline Bacteria & $27.2 \%(271)$ \\
\hline Fungi & $12.8 \%(128)$ \\
\hline Virus & $3.6 \%(36)$ \\
\hline
\end{tabular}


most frequent being mucus secretions (82.4\%), mucus plugs (17.6\%), haematic secretions/clots (23.7\%) and diffuse mucosal hyperaemia (11.4\%) (figure 1). The most frequent therapy consisted in atelectasis resolution or mucus aspiration (82.3\%). Among 147 bronchoscopies performed to rule out superinfection, the microbiological samples were obtained from: BAS (11.6\%), BAL (10.9\%), bronchial washing (52.5\%), and BAS in combination with BAL (21.7\%). The diagnostic yield was 42.9\%, including 71 microbiological isolations which are detailed as supplementary material.

\section{Impact of endoscopic findings on outcomes}

All patients with RT-PCR-confirmed COVID-19, either in nasopharyngeal swab or in lower respiratory tract specimens, were included to evaluate clinical, radiological and endoscopic features associated with mortality $(n=496)$. Univariate and multivariate logistic regression analyses to predict in-hospital mortality at 90 days are shown in table 3. The independent predictors of in-hospital mortality were: older age (OR 1.06, 95\% CI 1.03-1.08; $\mathrm{p}<0.001$ ), mucus plugs as indication for bronchoscopy (OR 1.60, 95\% CI 1.022.53; $\mathrm{p}=0.041$ ), absence of diffuse mucosal hyperaemia (OR 0.49, 95\% CI 0.25-0.97; p=0.041), and the presence of haematic secretions (OR 1.79, 95\% CI 1.05-3.05; $\mathrm{p}=0.032$ ) in the distal bronchial tract. A Charlson score $\geqslant 3$ was kept in the final model as clinically relevant information. The interval from hospital admission to bronchoscopy behaved as a confounding factor and was controlled in the final model. In the survival analysis, the presence of haematic secretions in the distal bronchial tract was the only endoscopic finding associated with mortality: $53.2 \%$ versus $35.7 \%$ at 60 days and $61 \%$ versus $39.5 \%$ at 90 days post-bronchoscopy (log-rank $\mathrm{p}=0.038$ ) (figure 2).

\section{Discussion}

The present study was carried out in the largest cohort published to date and provides key evidence regarding potential indications for bronchoscopy in patients with suspected or confirmed COVID-19, both for diagnostic or therapeutic purposes. Interestingly, some bronchoscopic findings were independently associated with in-hospital mortality after controlling for potential confounders. This information could be used to refine healthcare pathways and to reduce heterogeneity in clinical practice, in order to improve outcomes in patients with severe COVID-19.

The diagnosis of SARS-CoV-2 pneumonia is challenging when RT-PCR is negative in conventional nasopharyngeal swabs. Previous studies have suggested that lower respiratory tract specimens could increase sensitivity and allow diagnosis in patients with reduced viral load [3], while others recommend avoiding bronchoscopy for diagnostic purposes [14]. The selection of candidates for diagnostic bronchoscopy is paramount as this is an invasive procedure, not without risk of complications, and there is also a potential risk of spreading the infection to the medical staff due to the aerosols generated therein [15]. Only patients with high clinical suspicion of COVID-19 and typical radiological findings who test negative in two consecutive nasopharyngeal swabs may be considered for diagnostic bronchoscopy.
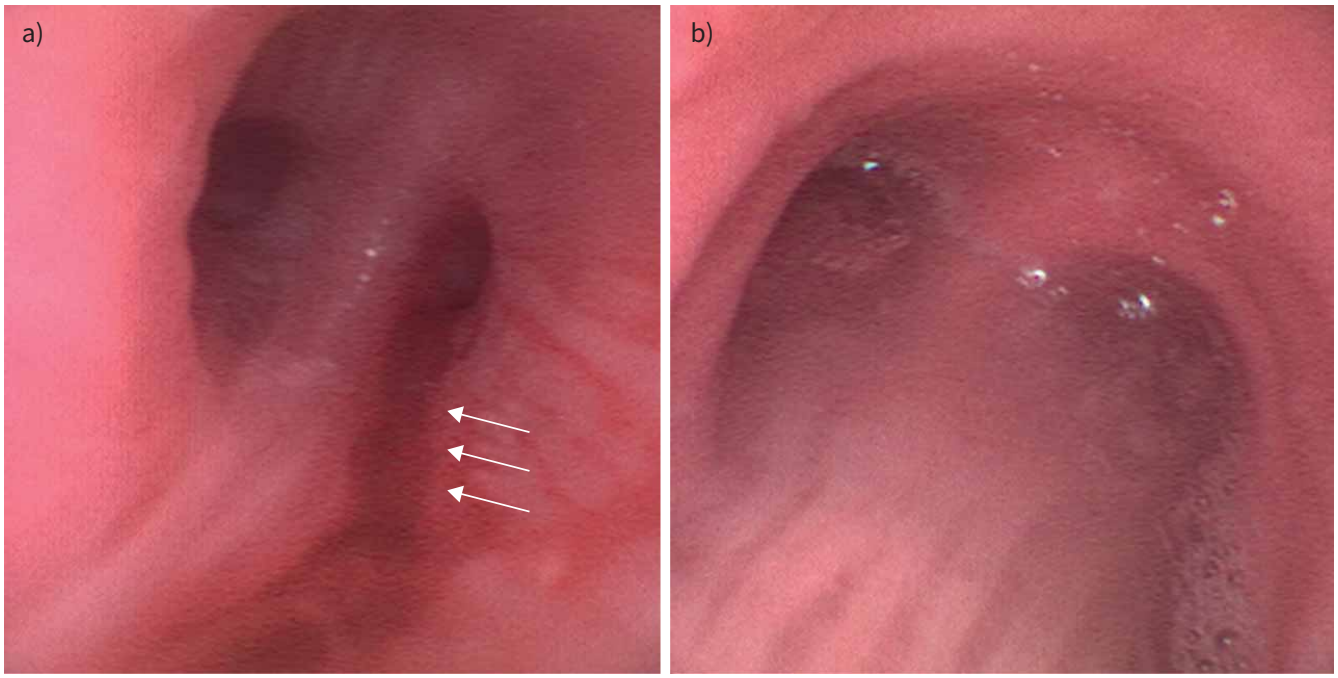

FIGURE 1 Most representative bronchoscopic findings in patients with RT-PCR-confirmed COVID-19. Haematic secretions (arrows). b) Mucus secretions. Pictures were obtained using disposable bronchoscopes. 


\begin{tabular}{|c|c|c|c|c|c|c|}
\hline \multirow[t]{2}{*}{ Variables } & \multicolumn{2}{|c|}{ Univariate analysis } & \multicolumn{2}{|c|}{$\begin{array}{l}\text { Multivariate analysis } \\
\quad \text { (initial model) }\end{array}$} & \multicolumn{2}{|c|}{$\begin{array}{l}\text { Multivariate analysis } \\
\text { (final model) }\end{array}$} \\
\hline & OR $(95 \% \mathrm{Cl})$ & p-value & OR $(95 \% \mathrm{Cl})$ & $p$-value & OR $(95 \% \mathrm{Cl})$ & $\mathrm{p}$-value \\
\hline Age & $1.05(1.03-1.08)$ & $<0.001$ & $1.05(1.03-1.08)$ & $<0.001$ & $1.06(1.03-1.08)$ & $<0.001$ \\
\hline Sex, women & $1.14(0.74-1.74)$ & 0.551 & & & & \\
\hline \multicolumn{7}{|l|}{ Medical history } \\
\hline Diabetes & $0.96(0.62-1.50)$ & 0.872 & & & & \\
\hline Hypertension & $1.22(0.84-1.77)$ & 0.292 & $0.93(0.59-1.47)$ & 0.768 & & \\
\hline Cardiovascular & $0.73(0.39-1.37)$ & 0.333 & & & & \\
\hline Bronchopulmonary & $1.08(0.64-1.83)$ & 0.767 & & & & \\
\hline Neoplasms & $0.77(0.40-1.47)$ & 0.427 & & & & \\
\hline Charlson comorbidity index $\geqslant 3$ & $1.15(0.63-2.09)$ & 0.644 & $1.25(0.62-2.53)$ & 0.526 & $1.07(0.56-2.04)$ & 0.834 \\
\hline Current/past smoking & $1.18(0.79-1.76)$ & 0.403 & & & & \\
\hline Interval hospital admission to FBC & $0.99(0.98-1.00)$ & 0.053 & $0.99(0.98-1.00)$ & 0.163 & $0.99(0.98-1.00)$ & 0.076 \\
\hline \multicolumn{7}{|l|}{ Clinical presentation } \\
\hline Fever & $0.84(0.51-1.37)$ & 0.491 & & & & \\
\hline Dyspnoea & $1.26(0.84-1.88)$ & 0.263 & $1.44(0.88-2.33)$ & 0.144 & & \\
\hline Cough & $0.82(0.54-1.25)$ & 0.361 & & & & \\
\hline Gastrointestinal & $1.05(0.67-1.62)$ & 0.832 & & & & \\
\hline Myalgias & $1.08(0.72-1.60)$ & 0.713 & & & & \\
\hline \multicolumn{7}{|l|}{ Laboratory parameters } \\
\hline Lymphocyte count & $1.00(0.99-1.00)$ & 0.272 & $1.00(1.00-1.00)$ & 0.901 & & \\
\hline D dimer & $1.00(1.00-1.00)$ & 0.068 & $1.00(1.00-1.00)$ & 0.123 & & \\
\hline Lactate dehydrogenase & $1.00(1.00-1.00)$ & 0.543 & & & & \\
\hline Ferritin & $1.00(1.00-1.00)$ & 0.318 & & & & \\
\hline C-reactive protein & $1.00(0.99-1.00)$ & 0.151 & $1.00(0.99-1.00)$ & 0.206 & & \\
\hline Interleukin-6 & $1.00(1.00-1.00)$ & 0.498 & & & & \\
\hline Radiograph, bilateral involvement & $1.11(0.67-1.84)$ & 0.681 & & & & \\
\hline \multicolumn{7}{|l|}{ Indications for bronchoscopy } \\
\hline Atelectasis & $1.02(0.53-1.96)$ & 0.951 & & & & \\
\hline Mucus plugs & $1.42(0.94-2.14)$ & 0.092 & $1.63(0.97-2.73)$ & 0.063 & $1.60(1.02-2.53)$ & 0.041 \\
\hline Haemoptysis & $1.26(0.60-2.67)$ & 0.540 & & & & \\
\hline Radiological & $1.41(0.91-2.21)$ & 0.123 & & & & \\
\hline \multicolumn{7}{|l|}{ Persistence/progression } \\
\hline Difficult mechanical ventilation ${ }^{\#}$ & $1.21(0.83-1.75)$ & 0.319 & & & & \\
\hline \multicolumn{7}{|l|}{ Bronchoscopy findings } \\
\hline Mucosal hyperaemia & $0.81(0.44-1.50)$ & 0.506 & $0.45(0.22-0.94)$ & 0.035 & $0.49(0.25-0.97)$ & 0.041 \\
\hline Thick mucus & $1.19(0.82-1.73)$ & 0.365 & $1.67(0.99-2.80)$ & 0.051 & & \\
\hline Fluid mucus & $0.96(0.61-1.52)$ & 0.964 & $1.42(0.75-2.67)$ & 0.281 & & \\
\hline Mucus plugs & $1.41(0.89-2.26)$ & 0.142 & $1.13(0.63-2.06)$ & 0.673 & & \\
\hline Haematic secretions & $1.78(1.09-2.89)$ & 0.020 & $1.98(0.63-2.06)$ & 0.028 & $1.79(1.05-3.05)$ & 0.032 \\
\hline Clots & $1.59(0.70-3.57)$ & 0.266 & $1.87(0.30-2.51)$ & 0.793 & & \\
\hline
\end{tabular}

Univariate and multivariate logistic regression analyses were used. FBC: fibreoptic bronchoscopy. ${ }^{\#}$ : includes impossible weaning from mechanical ventilation.

The diagnostic yield of lower respiratory tract samples in the present study was $36.7 \%$ for SARS-CoV-2 (53\% if alternative microbiological agents were considered), which was lower than in previous reports (55$71 \%)[3,5]$. This may be due to different selection criteria including the number of prior negative swabs and CT findings. In our study, patients with positive and negative results had a similar clinical presentation and laboratory findings, suggestive of high clinical suspicion of COVID-19 in this cohort. Gastrointestinal symptoms could identify a subgroup of candidates for diagnostic bronchoscopy. Another way to optimise the selection of candidates would be to avoid patients with atypical radiological findings [16]. According to our results, bilateral involvement in the chest radiography and typical or fairly typical findings in the CT as previously defined [11], may help to achieve better selection of patients, thus refining clinical pathways.

International scientific societies and expert panels have issued recommendations to safely perform bronchoscopy in patients with suspected or confirmed COVID-19 [17-20]. However, statements regarding the optimal approach to obtain microbiological samples are vague. This may explain the heterogeneity in 


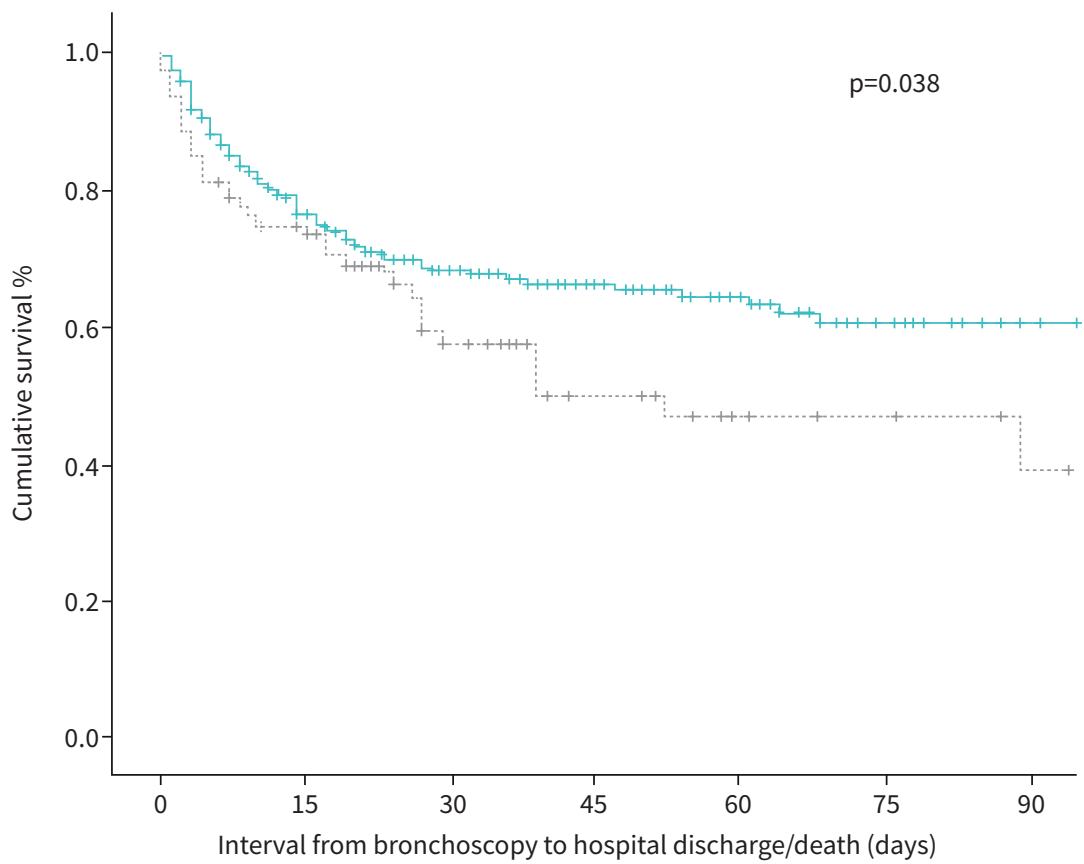

Group; number at risk

30 days
50

50

168

\begin{abstract}
60 days
\end{abstract}
10

57

90 days
5
24

FIGURE 2 Kaplan-Meier curve showing the influence of haematic secretions in the distal bronchial tract on mortality in 496 patients with RT-PCR-confirmed COVID-19 admitted to the hospital.

clinical practice, as illustrated in the present study. According to our results, BAS alone should be avoided but other options including BAL, bronchial washing or BAL in combination with BAS, would be equally valid. In contrast, guidelines are broadly homogeneous regarding protocols to protect healthcare personnel [17, 20, 21]. In brief, bronchoscopies in patients with suspected or confirmed COVID-19 should be performed in negative-pressurised or in adequately ventilated rooms. The involved healthcare personnel may be experienced and reduced to the minimum (two or three people depending on the procedure). Disposable bronchoscopes are advised. Individual enhanced third-degree protection elements are required (protective glasses or face shield, FFP3 face masks, protective clothing, gloves, etc.). Unfortunately, some of these recommendations are difficult to implement in real clinical practice, particularly in secondary hospitals, which were overwhelmed during the peak of the pandemic. Negative-pressurised rooms are anecdotal in ICUs where most therapeutic endoscopies need to be performed. These structural deficiencies should be urgently amended by healthcare authorities to protect medical staff from COVID-19 transmission. In any case, the decision to perform (or not perform) a bronchoscopy in a patient with COVID-19 should be taken after a careful weighing of potential benefits against the potential risk of disease transmission to healthcare personnel.

Critically ill patients with COVID-19 usually require prolonged mechanical ventilation. Bronchoscopy may help to prevent, diagnose or resolve ventilator-related complications. This is the first multicentre study describing the indications and procedures in this setting. The presence of mucus plugs was the only indication independently associated with worse outcomes (60\% increased mortality rates as compared with other indications), although it is tightly related to other indications such as atelectasis, superinfection and difficult mechanical ventilation. It is paramount to optimise ventilation to prevent excess secretions and to perform frequent aspirations through the endotracheal tube [20].

There are well established clinical, analytical and radiological predictors of poor outcomes in patients with COVID-19 including (but not limited to) older age, men, increased comorbidities, lymphopenia, increased $\mathrm{D}$ dimer and serum ferritin, and extent of pneumonia in the chest CT [22, 23]. This is the first study sufficiently powered to analyse the impact of bronchoscopic findings on outcomes among hospitalised patients with COVID-19. The presence of diffuse mucosal hyperaemia was associated with reduced 
in-hospital mortality rates, as it is likely a typical feature of an earlier phase of COVID-19, indicating acute inflammation [24]. This situation may still be reversible with or without anti-inflammatory drugs such as corticosteroids [25]. However, the disappearance of this endoscopic sign under persistent respiratory insufficiency may indicate a poor prognosis. The presence of haematic secretions in the distal bronchial tract was an independent predictor of increased in-hospital mortality. In contrast to diffuse mucosal hyperaemia, haematic secretions could translate into irreversible damage of the capillaries and the interstitial/alveolar space, which characterises the most advanced and severe forms of COVID-19 [26-28]. Indeed, the presence of haematic secretions identified a subgroup of very sick patients (16\%) with in-hospital mortality above $60 \%$. Further studies focused on this subpopulation are needed to delineate more aggressive and life-saving therapies.

The present study is limited by its ambispective design which precluded a protocolised clinical management of the study population. Although laboratory and radiological assessment of patients with COVID-19 varied among different institutions, making it difficult to extract solid conclusions regarding these parameters, the study adequately captured the heterogeneity in real clinical practice. On the other hand, the number of patients in the clinical suspicion cohort was limited as this indication is uncommon and not accepted by some experts [14]. Finally, a potential relationship between ventilator-derived trauma and some bronchoscopic findings in critically ill patients could not be ruled out.

In conclusion, bronchoscopy is pivotal as part of the armamentarium against COVID-19. In carefully selected patients with clinical and radiological suspicion of SARS-CoV-2 pneumonia who test negative in nasopharyngeal swabs, a lower respiratory tract specimen may provide an acceptable diagnostic yield, also including the identification of alternative microbiological agents or superinfection. In critically ill patients with COVID-19, bronchoscopy allows removal of mucus plugs and intrabronchial clots, and the resolution of atelectasis, thereby improving mechanical ventilation. Finally, haematic secretions in the respiratory tract and absence of diffuse mucosal hyperaemia are poor prognostic features.

Acknowledgements: We acknowledge Donna Pringle for professional English language polishing.

Data availability: Upon publication, data collected for the study will be made available for others in a Mendeley repository.

Conflict of interest: M. Arenas-De Larriva reports a travel grant from Novartis and lecture fees from Ferrer outside the submitted work. R. Martín-DeLeon has nothing to disclose. B. Urrutia Royo has nothing to disclose. I. Fernández-Navamuel has nothing to disclose. A. Gimenez Velando has nothing to disclose. L. Nuñez García has nothing to disclose. C. Centeno Clemente has nothing to disclose. F. Andreo García has nothing to disclose. A. Rafecas Codern has nothing to disclose. C. Fernández-Arias has nothing to disclose. V. Pajares Ruiz has nothing to disclose. A. Torrego Fernández has nothing to disclose. O. Rajas has nothing to disclose. G. Iturricastillo has nothing to disclose. R. Garcia Lujan has nothing to disclose. L. Comeche Casanova has nothing to disclose. A. Sánchez-Font has nothing to disclose. R. Aguilar-Colindres has nothing to disclose. R. Larrosa-Barrero has nothing to disclose. R. García García has nothing to disclose. R. Cordovilla has nothing to disclose. A. Núñez-Ares has nothing to disclose. A. Briones-Gómez has nothing to disclose. E. Cases Viedma reports lecture fees from Ambu outside the submitted work. J. Franco has nothing to disclose. J. Cosano Povedano reports a travel grant from Izasa scientific outside the submitted work. M.L. Rodríguez-Perálvarez reports lecture fees from Novartis, Astellas and Intercept outside the submitted work. J.J. Cebrian Gallardo has nothing to disclose. M. Nuñez Delgado has nothing to disclose. M. Pavón-Masa has nothing to disclose. M. Valdivia Salas has nothing to disclose. J. Flandes has nothing to disclose.

Support statement: The present study was funded by the Spanish Society of Pulmonology and Thoracic Surgery: extraordinary grant PII 2020 for research in COVID-19. Funding information for this article has been deposited with theCrossref Funder Registry.

References

1 Huang C, Wang Y, Li X, et al. Clinical features of patients infected with 2019 novel coronavirus in Wuhan, China. Lancet 2020; 395: 497-506.

2 Fennelly KP. Particle sizes of infectious aerosols: implications for infection control. Lancet Respir Med 2020; 8: 914-924.

3 Wang W, Xu Y, Gao R, et al. Detection of SARS-CoV-2 in different types of clinical specimens. JAMA 2020; 323: 1843-1844.

4 Lentz RJ, Colt H. Summarizing societal guidelines regarding bronchoscopy during the COVID-19 pandemic Respirology 2020; 25: 574-577. 
5 Mondoni M, Sferrazza Papa GF, Rinaldo R, et al. Utility and safety of bronchoscopy during the SARS-CoV-2 outbreak in Italy: a retrospective, multicentre study. Eur Respir J 2020; 56: 2002767.

6 Torrego A, Pajares V, Fernandez-Arias C, et al. Bronchoscopy in patients with COVID-19 with invasive mechanical ventilation: a single-center experience. Am J Respir Crit Care Med 2020; 202: 284-287.

7 Chang SH, Jiang J, Kon ZN, et al. Safety and efficacy of bronchoscopy in critically ill patients with coronavirus disease 2019. Chest 2021; 159: 870-872.

8 Kanne JP, Bai H, Bernheim A, et al. COVID-19 Imaging: what we know now and what remains unknown. Radiology 2021; 299: 204522.

9 Harris PA, Taylor R, Minor BL, et al. The REDCap consortium: building an international community of software platform partners. J Biomed Inform 2019; 95: 103208.

10 Charlson ME, Pompei P, Ales KL, et al. A new method of classifying prognostic comorbidity in longitudinal studies: development and validation. J Chronic Dis 1987; 40: 373-383.

11 Salehi S, Abedi A, Balakrishnan S, et al. Coronavirus disease 2019 (COVID-19) imaging reporting and data system (COVID-RADS) and common lexicon: a proposal based on the imaging data of 37 studies. Eur Radiol 2020; 30: 4930-4942.

12 Grasselli G, Zangrillo A, Zanella A, et al. Baseline characteristics and outcomes of 1591 patients infected with SARS-CoV-2 admitted to ICUs of the Lombardy region, Italy. JAMA 2020; 323: 1574-1581.

13 Bhatraju PK, Ghassemieh BJ, Nichols M, et al. COVID-19 in critically ill patients in the Seattle region - case series. N Engl J Med 2020; 382: 2012-2022.

14 Ora J, Puxeddu E, Cavalli F, et al. Does bronchoscopy help the diagnosis in COVID-19 infection? Eur Respir J 2020; 56: 2001619.

15 Jackson T, Deibert D, Wyatt G, et al. Classification of aerosol-generating procedures: a rapid systematic review. BMJ Open Respir Res 2020; 7: e000730.

16 Ai T, Yang Z, Hou H, et al. Correlation of chest CT and RT-PCR testing for coronavirus disease 2019 (COVID-19) in China: a report of 1014 cases. Radiology 2020; 296: E32-E40.

17 Wahidi MM, Shojaee S, Lamb CR, et al. The use of bronchoscopy during the coronavirus disease 2019 pandemic: CHEST/AABIP guideline and expert panel report. Chest 2020; 158: 1268-1281.

18 Luo F, Darwiche K, Singh S, et al. Performing bronchoscopy in times of the COVID-19 pandemic: practice statement from an international expert panel. Respiration 2020; 99: 417-422.

19 Steinfort DP, Herth FJF, Irving LB, et al. Safe performance of diagnostic bronchoscopy/EBUS during the SARS-CoV-2 pandemic. Respirology 2020; 25: 703-708.

20 Yang $\mathrm{H}$, Chen $\mathrm{H}$, Gao B, et al. Expert panel consensus statement on the applications and precaution strategies of bronchoscopy in patients with COVID-19. Endosc Ultrasound 2020; 9: 211-219.

21 Cordovilla R, Alvarez S, Llanos L, et al. SEPAR and AEER consensus recommendations on the use of bronchoscopy and airway sampling in patients with suspected or confirmed COVID-19 infection. Arch Bronconeumol 2020; 56: Suppl. 2, 19-26.

22 Zhou F, Yu T, Du R, et al. Clinical course and risk factors for mortality of adult inpatients with COVID-19 in Wuhan, China: a retrospective cohort study. Lancet 2020; 395: 1054-1062.

23 Colombi D, Villani GD, Maffi G, et al. Qualitative and quantitative chest CT parameters as predictors of specific mortality in COVID-19 patients. Emerg Radiol 2020; 27: 701-710.

24 Batah SS, Fabro AT. Pulmonary pathology of ARDS in COVID-19: a pathological review for clinicians. Respir Med 2021; 176: 106239.

25 Cano EJ, Fuentes XF, Campioli CC, et al. Impact of corticosteroids in coronavirus disease 2019 outcomes: systematic review and meta-analysis. Chest 2021; 159: 1019-1040.

26 Fox SE, Akmatbekov A, Harbert JL, et al. Pulmonary and cardiac pathology in African American patients with COVID-19: an autopsy series from New Orleans. Lancet Respir Med 2020; 8: 681-686.

27 Bradley BT, Maioli H, Johnston R, et al. Histopathology and ultrastructural findings of fatal COVID-19 infections in Washington State: a case series. Lancet 2020; 396: 320-332.

28 Menter T, Haslbauer JD, Nienhold R, et al. Postmortem examination of COVID-19 patients reveals diffuse alveolar damage with severe capillary congestion and variegated findings in lungs and other organs suggesting vascular dysfunction. Histopathology 2020; 77: 198-209. 